\title{
Maternal Endotoxin Exposure Results in Abnormal Neuronal Architecture in the Newborn Rabbit
}

\author{
Bindu Balakrishnan $^{a, b}$ Hui Dai ${ }^{b}$ James Janisse ${ }^{c}$ Roberto Romero $^{d}$ \\ Sujatha Kannan ${ }^{\text {a, b }}$ \\ ${ }^{a}$ Department of Anesthesiology and Critical Care Medicine, Johns Hopkins University, Baltimore, Md., ${ }^{b}$ Department \\ of Pediatrics, Children's Hospital of Michigan, Wayne State University School of Medicine, 'Department of \\ Family Medicine and Public Health Sciences, Detroit, Mich., and d Perinatology Research Branch, NICHD, NIH, DHHS, \\ Detroit, Mich., and Bethesda, Md., USA
}

\section{Key Words}

Maternal intrauterine inflammation · Dendrites · Dendritic spine $\cdot$ Cerebral palsy $\cdot$ Neuronal injury $\cdot$ Perinatal brain injury $\cdot$ Thalamus

\begin{abstract}
Maternal intrauterine inflammation/infection is a potential risk factor for the development of neurologic disorders such as cerebral palsy (CP) in preterm and term infants. CP is associated with white matter and grey matter injury. In the current study, we used a rabbit model of $C P$ in which pregnant rabbits are administered intrauterine injections of the endotoxin lipopolysaccharide. We then investigated the extent of neuronal damage in the newborn kit brain. We observed an overall decrease in the number of MAP2-stained neurons and an increase in Fluoro-Jade C-stained cells in the anterior thalamus of 1-day-old rabbit brain. We also observed an overall decrease in the number of branching points and spine density in the retrosplenial cortex, a major output region of the anterior thalamus that is involved in cognition and memory. The loss of spines and dendritic atrophy in the
\end{abstract}

retrosplenial cortex may be caused by loss of presynaptic input from the thalamus. Our study indicates that the cognitive impairments seen in patients with CP may be related to the degeneration of neurons and abnormal arborization of the thalamic and cortical neurons.

(c) 2013 S. Karger AG, Basel

\section{Introduction}

Intrauterine infection during pregnancy is associated with an increased risk for the development of a wide spectrum of neurologic disorders, such as cerebral palsy (CP), autism, and schizophrenia, in the offspring [1-3]. $\mathrm{CP}$, which results from insult to the developing brain, is associated with motor deficits as well as sensory and cognitive disorders. Thus, CP not only involves injury to the white matter tracts but also to the grey matter. Neuronal injury has been noted in the cerebral cortex, hippocampus, subiculum, pons, and cerebellum of postmortem brain specimens from patients with periventricular leukomalacia (PVL) [4-7]. Maternal infection near term

\section{KARGER}

E-Mail karger@karger.com

www.karger.com/dne
(C) 2013 S. Karger AG, Basel

0378-5866/13/0355-0396\$38.00/0
Dr. Sujatha Kannan, MD

Anesthesiology and Critical Care Medicine

Johns Hopkins University School of Medicine, Kennedy Krieger Institute

1800 Orleans St, Baltimore, MD (USA)

E-Mail skannan3@jhmi.edu 
can trigger perinatal inflammation via activated microglia. Because activated microglia are found in large densities in the white matter tracts during the second and third trimesters [8], an immune activation during this period can inflict a deleterious effect on the developing brain by changing the microglial function and altering the balance between neuroprotection and neurodegeneration [9].

In our model of $\mathrm{CP}$, we have reported a substantial increase in activated microglial cells in the white matter regions of rabbit kit brains after exposure to maternal intrauterine lipopolysaccharide (LPS) injection at gestational age 28 days $[10,11]$. The increase in activated microglia correlated with a decrease in myelin basic protein and increase in motor deficits in these kits [10]. We have also shown that maternal endotoxin exposure results in decreased cortical serotonin concentration and loss of serotonin-immunoreactive axons in the somatosensory cortex of newborn rabbit kits [12]. Serotonin can regulate processes such as neurogenesis, apoptosis, axon branching, and dendritogenesis and is thought to exert a neurotrophic effect [13]. Early postnatal depletion of serotonin has been shown to reduce the length of dendrites and the number of dendritic spines in the hippocampal neurons [14].

In this study, we hypothesized that maternal endotoxin exposure would result in loss of thalamic neurons, decreased arborization and injury to cortical neurons in the newborn rabbits. The thalamus is one of the brain regions most commonly affected in patients with CP [15-17]. It is an important relay station that coordinates all sensory signals before they are transmitted to the cortex. Atrophy of the thalamus has been described in patients with PVL [6]. We evaluated the neuronal injury in the anterior thalamus because this region is involved in learning and memory, and damage to it could be vital in the pathogenesis of subsequent cognitive impairment in patients with $\mathrm{CP}$. The anterior nuclei of the thalamus project mainly to the retrosplenial cortex (RSC), which plays a critical role in cognition and memory. The cortical plate of the retrosplenium, which is located between the hippocampus and neocortex, has afferent and efferent connections to the anterior thalamus. The RSC integrates information received from hippocampus, visual cortex, and anterior thalamus [18] and codes for cognitive functions [19]. RSC hypoactivity has been reported in patients with Alzheimer disease and diencephalic amnesia [20-22]. Damage to the RSC is sufficient to induce cognitive deficits, whereas lesions in the anterior thalamus amplify the impact of impairments of the RSC [21, 23-26].

Maternal Inflammation and Neuronal Injury in Rabbit Kits

\section{Materials and Methods}

\section{Animal Model}

New Zealand white rabbits with timed pregnancies were obtained from Covance Inc. (Covance Research Products Inc., Kalamazoo, Mich., USA). Pregnant rabbits underwent laparotomy at 28 days' gestation (term pregnancy $=31$ days) under general anesthesia (2-3\% isoflurane by mask) as previously described [10-12, $27,28]$. Briefly, $1 \mathrm{ml}$ of saline solution (control-saline) or $1 \mathrm{ml}$ of saline containing $20 \mu \mathrm{g} / \mathrm{kg}$ LPS (endotoxin; Escherichia coli serotype O127:B8, Sigma-Aldrich, St. Louis, Mo., USA) was injected along the length of the uterus between the fetuses, as previously described $[10-12,27,28]$. A third group consisted of rabbits that had no surgical intervention (control-no intervention). All kits were born spontaneously on gestational day 31 . All surgical procedures were approved by the Institutional Animal Care and Use Committee. A total of 15 pregnant rabbits and 42 kits were used for the study. Each group was composed of 5 pregnant rabbits with 3-4 kits from each litter that was randomly distributed for immunostaining, Golgi staining or Western blot analysis. All the kits underwent neurobehavioral testing as previously described for rabbits $[10,29,30]$.

\section{Immunostaining}

On day 1 of life, kits from the control-no intervention, controlsaline, and endotoxin groups (4 kits/group) were euthanized and perfused with $10 \%$ formalin. The brains were post-fixed, cryoprotected and cut into $30-\mu \mathrm{m}$ coronal sections and mounted on polyL-lysine-coated slides (Sigma-Aldrich). The thalamus was identified at the level of the dorsal hippocampus, where there is clear demarcation of anterior, medial and lateral nuclei as indicated in figure 1a. For quantification of MAP2-stained neurons in the anterior thalamus, 5 coronal sections, $90 \mu \mathrm{m}$ apart were used. The sections were stained overnight with mouse anti-MAP2 (1:100; Covance, Emeryville, Calif., USA) at $4^{\circ} \mathrm{C}$ and then treated with biotinylated secondary goat anti-mouse IgG (1:200; Vector, Burlingame, Calif., USA) at room temperature followed by Avidin biotin complex. Color was developed by using 3,3'-diaminobenzidine (Sigma-Aldrich).

\section{Fluoro-Jade C Stain}

Coronal sections from the three groups ( $\mathrm{n}=4 \mathrm{kits} /$ group, $5 \mathrm{sec}$ tions per kit) were stained with Fluoro-Jade $\mathrm{C}$ according to the manufacturer's instructions. Briefly, sections were immersed in alcohol containing $1 \%$ sodium hydroxide in $80 \%$ ethanol for $5 \mathrm{~min}$, rinsed for $2 \mathrm{~min}$ in $70 \%$ alcohol followed by $2 \mathrm{~min}$ in distilled water, and then incubated in $0.06 \%$ potassium permanganate solution for $20 \mathrm{~min}$. The slides were transferred to a $0.00005 \%$ solution of Fluoro-Jade C in $0.01 \%$ acetic acid for $10 \mathrm{~min}$. For each section, four separate fields at $\times 40$ magnification of the anterior thalami were examined, and the number of Fluoro-Jade C-positive cells was counted.

\section{MAP-2 Cell Count in the Thalamus by Stereology}

To obtain an unbiased estimate of the total number of MAP2positive neurons in the anterior thalamus, we used an optical fractionator probe (Stereo Investigator, MicroBrightfield Inc., Williston, Vt., USA) [31]. The cells stained with anti-MAP2 antibody were visualized under a $\mathrm{BH} 2$ Olympus light microscope (Olympus Optical Co. GmbH, Hamburg, Germany) attached to a motorized

Dev Neurosci 2013;35:396-405 

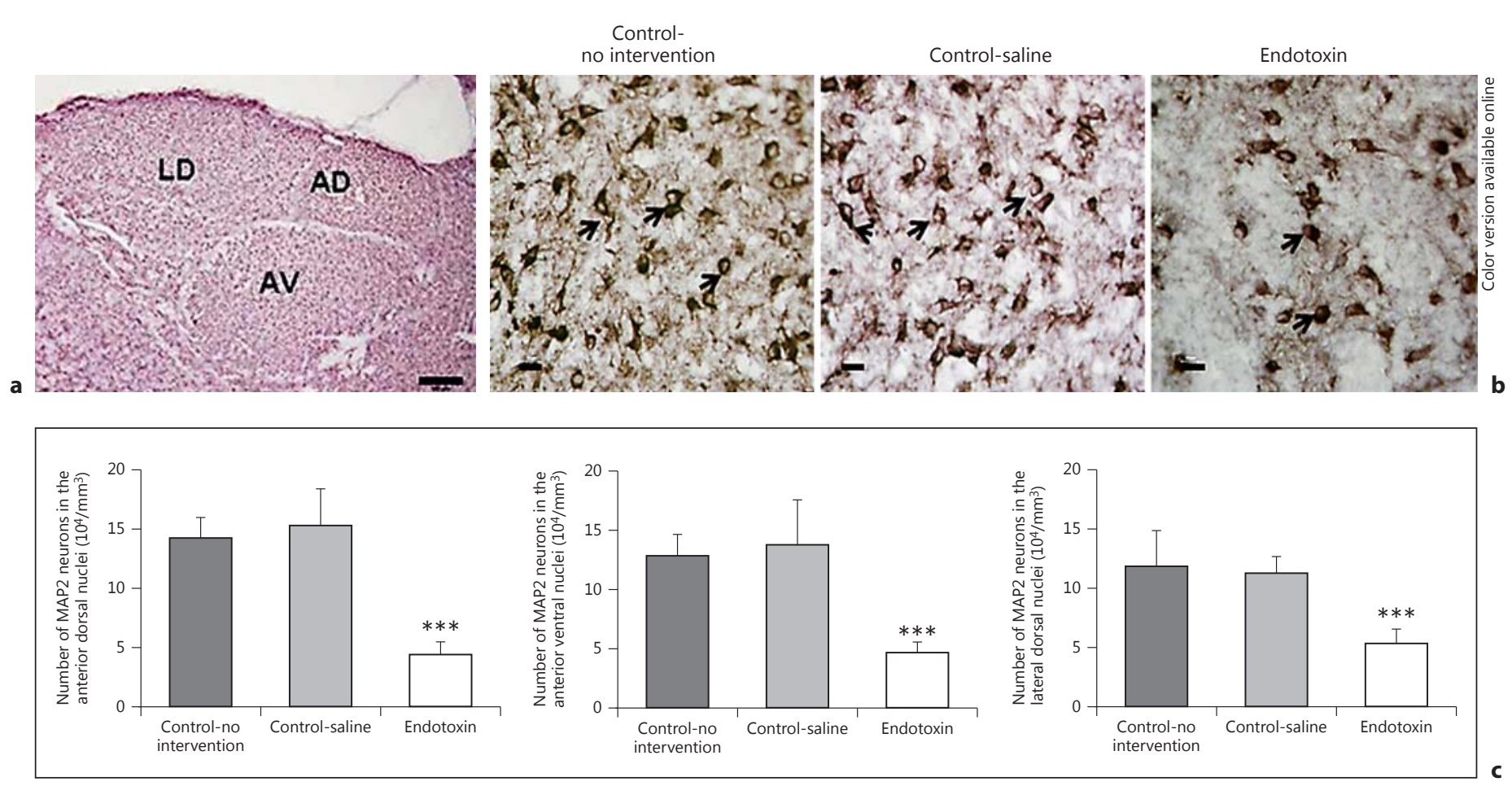

Fig. 1. MAP2-stained neurons in the anterior thalamus of brains from 1-day-old rabbits. a MAP2 staining in the anterior dorsal $(\mathrm{AD})$, anterior ventral (AV), and lateral dorsal (LD) nuclei of the anterior thalamus. Scale bar $=100 \mu \mathrm{m}$. b Representative images of MAP2-stained neurons in the anterior thalamus of 1-day-old kits born to mothers exposed to endotoxin or saline or who had no in- tervention. Arrows indicate MAP2-stained neurons. Scale bars = $10 \mu \mathrm{m}$. c The number of MAP2-stained neurons was significantly less in endotoxin-exposed kits than in kits from the two control groups. ${ }^{* * *} \mathrm{p}<0.001$, the endotoxin group was significantly different from the control groups (4 kits/group). stage and a stage encoder. The boundary of anterior dorsal, anterior ventral, and lateral dorsal nuclei was outlined as in figure 1a in each section under $\times 4$ magnification by drawing a contour. The sections were further visualized under $\times 40$ magnification with a sampling grid (area sampling fraction $=150 \times 150 \mu \mathrm{m}^{2}$ ) and systematic uniform random sampling $(x=315.2$ and $y=353.9)$. The average section thickness ranged between 8 and $10 \mu \mathrm{m}$, and the guard zone was calculated as $1 \%$. The total number of cells was determined by the formula

$$
N=\sum Q^{-} \frac{t}{h} \frac{1}{a s f} \frac{1}{s s f}
$$

where $\Sigma \mathrm{Q}^{-}$is the total number of neurons counted, $\mathrm{t}$ is the mean section thickness, $\mathrm{h}$ is the height of the optical dissector, asf is the area sampling fraction, and ssf is the section sampling fraction.

\section{Golgi Stain and Sholl's Analysis}

Kits from all three groups ( 4 kits/group) were sacrificed on day one of life. The brains were removed and processed for Golgi Cox staining (supplied by FD Neurotechnologies Inc., Ellicott City, Md., USA) as $120-\mu \mathrm{m}$ coronal sections. All slides were coded to prevent experimenter bias. The cortical neurons were viewed at random with a Leica DM 2500 microscope (Leica Microsystems, Wetzlar, Germany). All coronal brain sections from the level of bregma to the dorsoventral hippocampus were used. The RSC was identified as the cortical region close to the midline and above the corpus callosum (see fig. 3a) [32]. We selected 8-10 neurons in each kit that were dark, consistently impregnated with silver throughout the extent of all the dendrites, and relatively isolated from neighboring impregnated neurons. Neurons with truncated branches were omitted. Those cortical neurons that fulfilled the criteria were drawn by using camera lucida at $\times 40$ magnification. We quantified and analyzed dendritic branching points and dendritic intersections using Sholl's analysis [33]. We drew concentric circles that were $50 \mu \mathrm{m}$ apart on tracing paper using a stage micrometer scale at the same magnification at which the neurons were drawn. The center of the cell body of the neuron was placed at the center of the circle as the reference point. Dendritic intersections and branching points were measured from the soma by calculating values in each successive concentric segment. The dendritic branches and intersections were studied up to a length of 250 $\mu \mathrm{m}$ from the center of the soma.

\section{Spine Quantification}

Spines from the primary dendrites of the cortical neurons were visualized under an oil immersion objective (Leica immersion oil, Leica Microsystems) on an Olympus BX51 microscope (Olympus, Melville, N.Y., USA) and analyzed with Neurolucida image analy- 
Table 1. Behavioral scoring of the control-no intervention, control-saline and endotoxin rabbit kits on day 1 of life

\begin{tabular}{lllll}
\hline Outcome & Group & & p value \\
\cline { 2 - 4 } & $\begin{array}{l}\text { control-no } \\
\text { intervention } \\
(\mathrm{n}=10)\end{array}$ & $\begin{array}{l}\text { control-saline } \\
(\mathrm{n}=6)\end{array}$ & $\begin{array}{l}\text { endotoxin } \\
(\mathrm{n}=13)\end{array}$ & \\
\hline Locomotion steps & $2.90(2.6-3.2)$ & $3.00(2.2-3.8)$ & $1.54(0.9-2.2)$ & 0.005 \\
Head movement & 3.00 & $2.83(2.6-3.1)$ & $1.85(1.2-2.5)$ & 0.004 \\
Forelimb movement & 3.00 & $2.83(2.6-3.1)$ & $2.08(1.3-2.8)$ & 0.062 \\
Hindlimb movement & 3.00 & $2.50(1.9-3.1)$ & $1.38(1.0-1.8)$ & 0.003 \\
Righting reflex & 3.00 & $2.83(2.5-3.2)$ & $2.00(1.8-2.2)$ & $<0.001$ \\
Suck and swallow & 3.00 & 3.00 & $2.69(2.4-2.9)$ & \\
\hline
\end{tabular}

The endotoxin kits had significant impairments in locomotion and righting reflex compared to the control groups. Values represent mean $(95 \% \mathrm{CI})$. p values are calculated and reported for the control-saline vs. endotoxin groups where there is variation in the scores within the group.

sis software 6.21.2 (Microbrightfield). Five primary apical dendrites were selected randomly from the main shaft of cortical neurons along a distance of 50-250 $\mu \mathrm{m}$. All protrusions that had direct contact with the primary dendrite and were within a distance of 50 $\mu \mathrm{m}$ were counted as spines. The $50-\mu \mathrm{m}$ length was further divided into five segments of $10 \mu \mathrm{m}$ along the primary apical dendrites.

\section{Western Blotting}

Brain tissue lysates from cortex (40 mg protein) were prepared by standard techniques and separated on 12\% NuPage Novex BisTris MiniGels for Western blot analysis (XCell SureLock Mini-Cell, Invitrogen, Carlsbad, Calif., USA). Protein was transferred and immunoprobed according to previously described electrophoretic transfer and blotting methods (Invitrogen XCell II ${ }^{\mathrm{TM}}$ Blot Module). Membranes were probed with mouse anti-synaptophysin primary antibody (1:200 dilutions, Synaptic Systems GmbH, Germany). Horseradish peroxidase-conjugated secondary antibody (1:500, Abcam, Cambridge, Mass., USA) was used for detection. Blots were developed by using the enhanced chemiluminescence system (Invitrogen Western Breeze ${ }^{\circledR}$ Immunodetection Kit, USA) and X-ray photographic film (Eastman Kodak, New York, N.Y., USA). Expression of the housekeeping gene $\beta$-actin (Abcam) was used as an internal control for equal sample loading. In addition, developed films were analyzed semiquantitatively by densitometry with Image software (from the NIH). Protein expression was measured in optical density units and normalized to the corresponding expression of $\beta$-actin. Relative fold differences between untreated and treated samples were calculated by using these normalized values. Each group consisted of 5 kits from 5 different litters.

\section{Statistics}

Kits from the same litter were nested to account for the lack of independence, and differences across groups were analyzed by using generalized linear model equation (GEE) for (1) the number of branches, (2) spine density, (3) number of cells in anterior dorsal, anterior ventral, and lateral dorsal nuclei of thalamus, and (4) Fluoro-Jade-positive cells. The control-saline group that had $2 \mathrm{kits}$ from the same litter for the Golgi analysis, while the control-no interven- tion and endotoxin groups had kits from different litters. For immunostaining and Western blot, the kits in all groups were from different litters. For neurobehavioral analysis, we used Omnibus test for difference across all three groups, and when there was no variation in scores for a group, group differences with that group were based on $95 \%$ confidence interval (CI). If $95 \%$ CI does not contain the score for the group with no variation, this indicates significance at an alpha of 0.05 . We used one-way ANOVA followed by Tukey's post hoc test to analyze the difference in synaptophysin expression across groups. All values are presented as mean, 95\% CI.

\section{Results}

\section{Neurobehavior Scoring}

The newborn kits were scored for their motor ability by using a modified observational scoring system from video recordings as described previously $[10,29,30]$. The control-no intervention and control-saline kits had a significantly higher locomotion score when compared to the endotoxin kits $(\mathrm{p}=0.005)$. A significant difference was also observed between head movement and hind limb movement in endotoxin versus control groups $(\mathrm{p}=0.004$ and 0.003 ). The control groups had better righting reflex scores ( $\mathrm{p}<0.001$ for endotoxin vs. control-saline) and greater coordination of suck and swallow (table 1 ).

\section{MAP2-Stained Neurons Are Decreased and \\ Fluoro-Jade C-Stained Neurons Are Increased in the \\ Anterior Thalamus of Endotoxin-Exposed Kits}

Analysis with stereology revealed that the number of neurons in the anterior dorsal, anterior lateral, and anterior ventral regions of the thalamus was significantly re- 

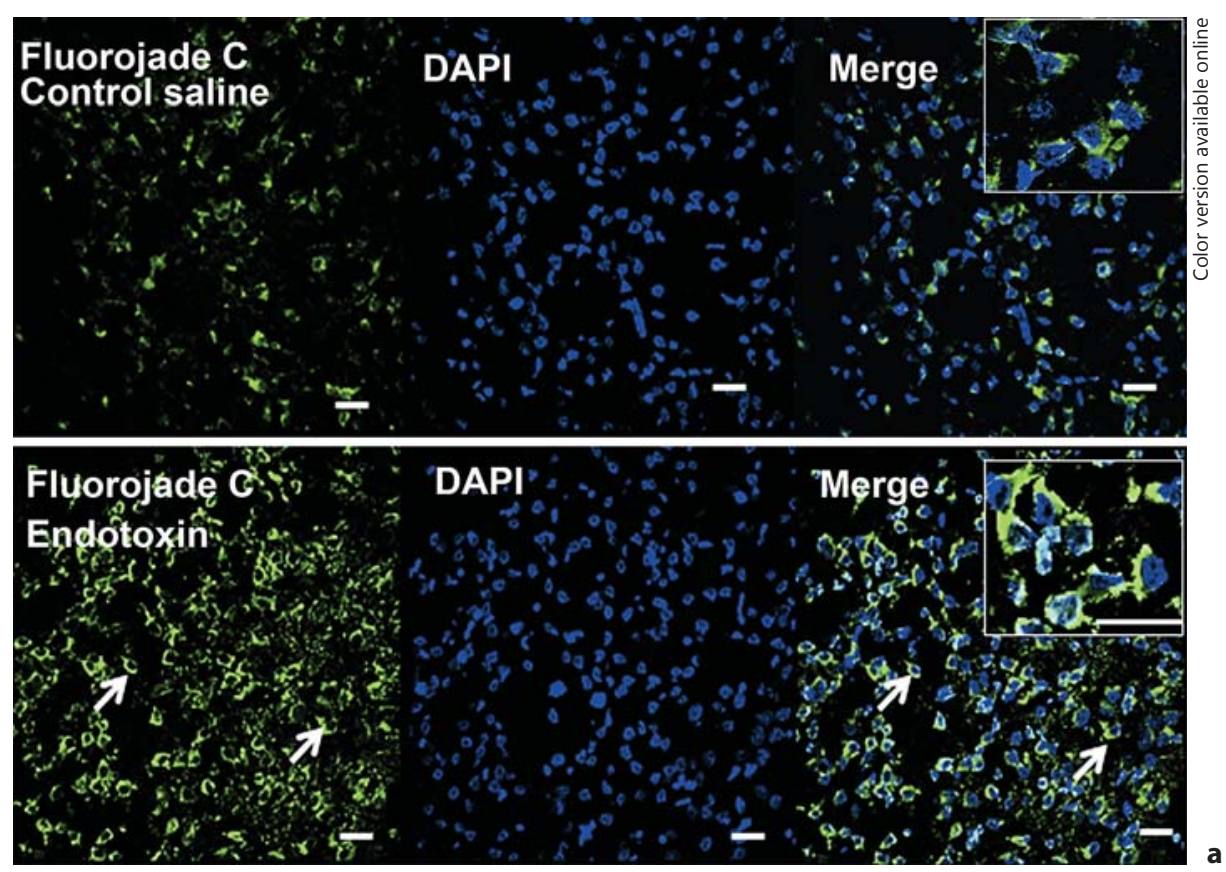

Fig. 2. Fluoro-Jade C staining for the detection of degenerating neurons in the anterior thalamus of 1-day-old rabbit kits. a Representative image of Fluoro-Jade Cstained degenerating neurons in the anterior thalamus of control-saline and endotoxin-exposed kits. Insets and arrows indicate degenerating neurons. Scale bars $=25$ $\mu \mathrm{m}$. b Quantification showed that the number of degenerating neurons was significantly greater in the endotoxin-exposed kits than in the saline-exposed kits. ${ }^{* * *} \mathrm{p}<$ 0.001 , the endotoxin group was significantly different from the control-saline group (4 kits/group).

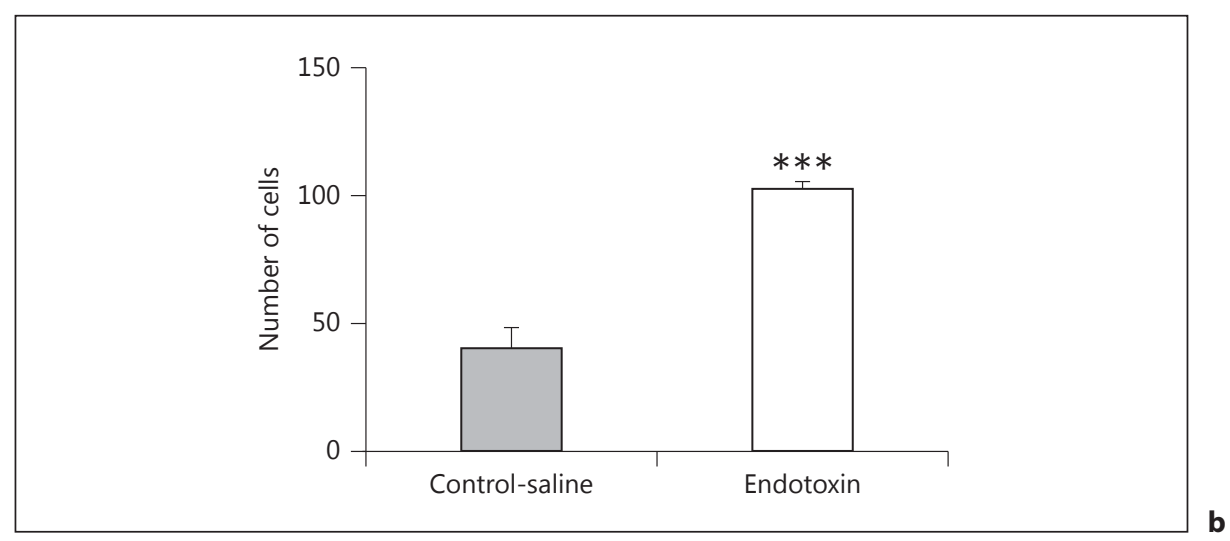

duced in the endotoxin group compared to the numbers in the control-no intervention and control-saline groups $(\mathrm{p}<$ 0.001; fig. 1). In the anterior dorsal nuclei, the endotoxin group had $4.3 \times 10^{4}$ cells $/ \mathrm{mm}^{3}\left(95 \% \mathrm{CI}: 3.2-5.5 \times 10^{4}\right) \mathrm{com}$ pared to $14.3 \times 10^{4}$ cells $/ \mathrm{mm}^{3}$ in the control-no intervention group (95\% CI: $\left.12.5-18.0 \times 10^{4}\right)$ and $15.3 \times 10^{4}$ cells/ $\mathrm{mm}^{3}$ (95\% CI: $\left.12.2-18.4 \times 10^{4}\right)$ in the control-saline group ( $p<0.001$ for comparison between endotoxin group and each control group). A similar decrease was observed in the anterior ventral and lateral dorsal nuclei of the thalamus in the endotoxin group compared to the control-no intervention and control-saline groups ( $\mathrm{p}<0.001$ for anterior ventral; $\mathrm{p}<0.001$ for lateral dorsal). The endotoxin group had $4.7 \times 10^{4}$ cells $/ \mathrm{mm}^{3}$ in the anterior ventral nuclei compared to $12.9 \times 10^{4}$ cells $/ \mathrm{mm}^{3}$ in the control-no intervention group and $13.8 \times 10^{4}$ cells $/ \mathrm{mm}^{3}$ in the control-saline group ( $\mathrm{p}<0.001$ for control-no intervention vs. endotoxin; $\mathrm{p}<$ 0.001 for control-saline vs. endotoxin). In the lateral dorsal nuclei, the endotoxin group had $5.4 \times 10^{4}$ neurons $/ \mathrm{mm}^{3}$ (95\% CI: $4.1-6.6 \times 10^{4}$ neurons $/ \mathrm{mm}^{3}$ ), whereas the control-no intervention group had $11.9 \times 10^{4}$ neurons $/ \mathrm{mm}^{3}$ (95\% CI: 8.9-15.0 $\times 10^{4}$ neurons $\left./ \mathrm{mm}^{3} ; \mathrm{p}<0.001\right)$ and the control-saline group had $11.3 \times 10^{4}$ neurons $/ \mathrm{mm}^{3}(95 \% \mathrm{CI}$ : 9.9-12.7 $\times 10^{4}$ neurons $\left./ \mathrm{mm}^{3} ; \mathrm{p}<0.001\right)$.

To determine whether the decreased number of neurons in the anterior thalamus of endotoxin-exposed kits was associated with degeneration of neurons, we used Fluoro-Jade $\mathrm{C}$, which stains basic proteins present in degenerating neurons. The number of Fluoro-Jade Cstained neurons was significantly higher in the anterior 
thalamus of endotoxin-exposed kits (mean, 102.9; 95\% CI: 99.5-106.2) than in that of kits in the saline-control group (mean, 41; 95\% CI: 33-49; p < 0.001; fig. 2).

\section{Dendritic Atrophy in the RSC of One-Day-Old Kits after Maternal Intrauterine Inflammation}

Newborn rabbit kits exposed to maternal intrauterine inflammation had fewer primary apical branches in their RSC than in control kits. GEE analysis showed a significant effect of endotoxin on dendritic arborization in the RSC of kits exposed to endotoxin compared to the control groups ( $\mathrm{p}<0.001)$. The total number of branches in the endotoxin group (7.9; 95\% CI: 7.2-8.6) was significantly less than that in the control-no intervention $(12.6 ; 95 \%$ CI: $11.3-13.9)$ and control-saline groups (13.4; $95 \%$ CI: $12.3-14.5 ; \mathrm{p}<0.001)$. Thus, the number of dendritic branches was reduced in the endotoxin-exposed kits by $37 \%$ compared to the control-no intervention group and by $40 \%$ compared to the control-saline group (fig. 3 ).

\section{Maternal Intrauterine Inflammation Results in}

Decreased Spine Density in the Apical Branches of

\section{Retrosplenial Cortical Neurons}

GEE analysis revealed a significant reduction in the number of spines in the kits subjected to intrauterine maternal inflammation (mean, 39.9; 95\% CI: 35.9-42.3) compared to the number in control-no intervention and control-saline groups ( $\mathrm{p}<0.001$; fig. 4$)$. The endotoxin group had significantly fewer spines (mean, 39.9; 95\% CI: 35.9-42.3) than did the control-no intervention group (mean, 64.7; 95\% CI: 51.7-77.8; $\mathrm{p}<0.001$ ) or the controlsaline group (mean, 67.8; 95\% CI: 57.6-78.1; $<<0.001$ ). Thus, the number of spines in 1-day-old endotoxin-exposed kits was reduced by $38 \%$ compared to that in the control-no intervention group and by $41 \%$ compared to that in the control-saline group (fig. 4).

Fig. 3. Maternal intrauterine inflammation leads to a decreased number of apical dendrites in the RSC of 1-day-old rabbit kits. a Representative images of Golgi Cox-stained neurons in the RSC of the control-no intervention, control-saline, and endotoxin-exposed groups. The endotoxin-exposed kits had fewer apical branches than did kits in either control group. b Sholl's analysis revealed a significant difference between the endotoxin and the control groups in the number of dendritic branches along a distance of $250 \mu \mathrm{m} .{ }^{* * *} \mathrm{p}<0.001$, endotoxin vs. control groups (4 kits/ group).

Maternal Inflammation and Neuronal Injury in Rabbit Kits
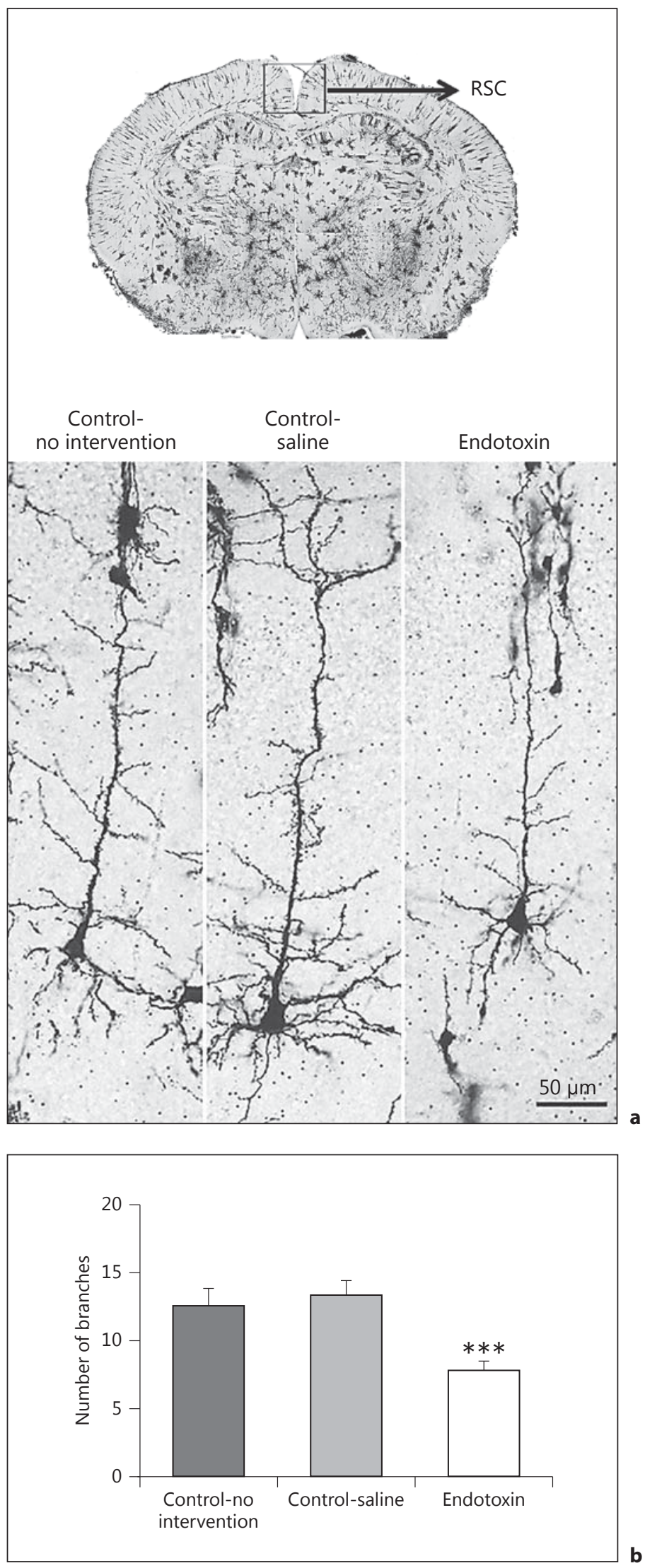

Dev Neurosci 2013;35:396-405 DOI: $10.1159 / 000353156$ 

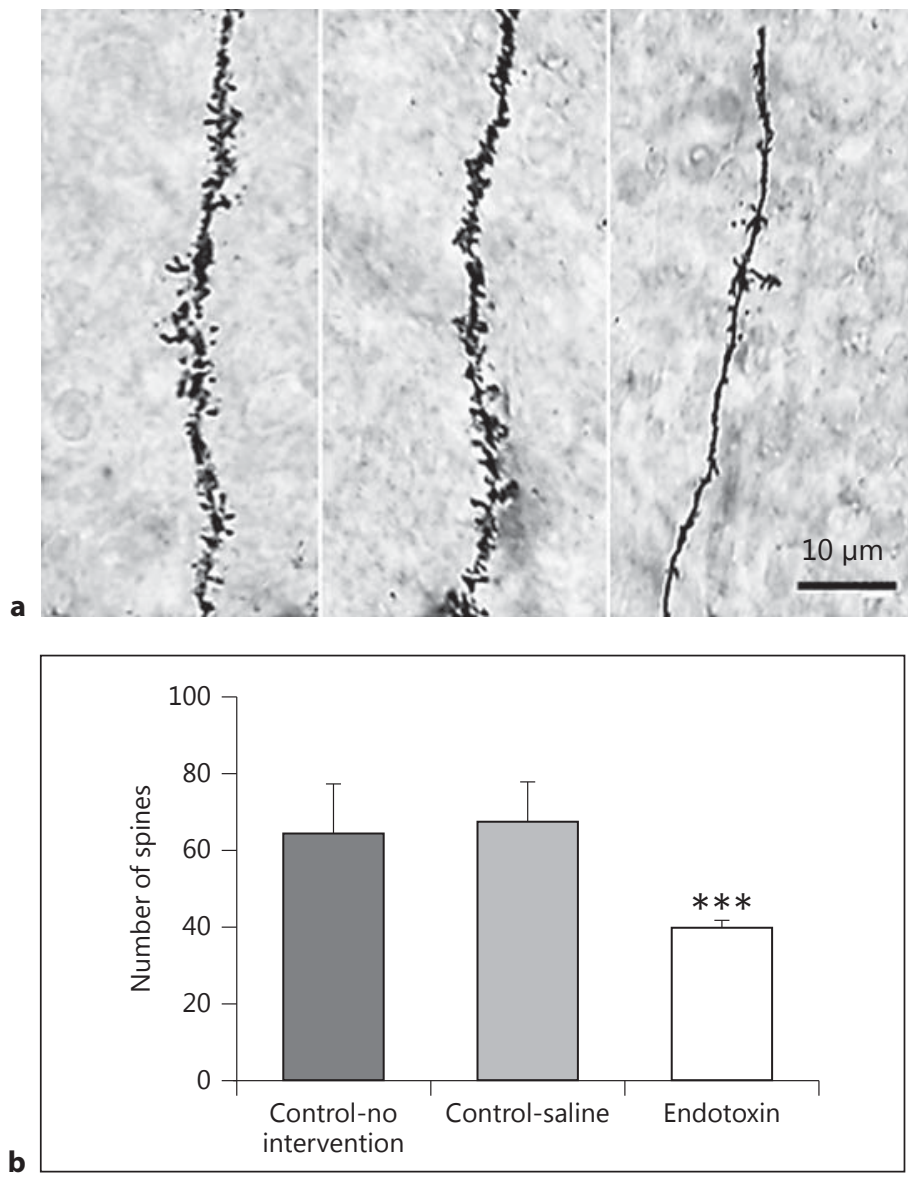

Fig. 4. Maternal intrauterine inflammation leads to a decreased number of spines in the apical branches of retrosplenial cortical neurons of 1-day-old rabbit kits. a Photomicrographs of Golgi Cox-impregnated spines in the primary apical branches of retrosplenial cortical neurons in the control-no intervention, controlsaline, and endotoxin-exposed groups. $\mathbf{b}$ The number of spines in the primary apical branches of the endotoxin-exposed group was significantly less along a distance of $50 \mu \mathrm{m}$ than in the control-no intervention and control-saline groups. ${ }^{* * *} \mathrm{p}<0.001$, endotoxin vs. control groups (4 kits/group).

\section{Decreased Synaptophysin Expression in the Cortex of}

Endotoxin-Exposed Kits

Synaptophysin is the most abundant synaptic vesicle protein, and is widely used as a marker for the presynaptic terminal. Synaptophysin is synthesized in the neuronal soma and transported along the axon to the neuron terminals [34]. Loss of thalamic neurons would result in decreased formation and transport of synaptophysin to the presynaptic terminals in the RSC. Decreased synaptophysin indicates a loss of input to the RSC dendrites leading to the reduction in the number of branches and spines in the retrosplenial cortical neurons in endotoxin kits. In
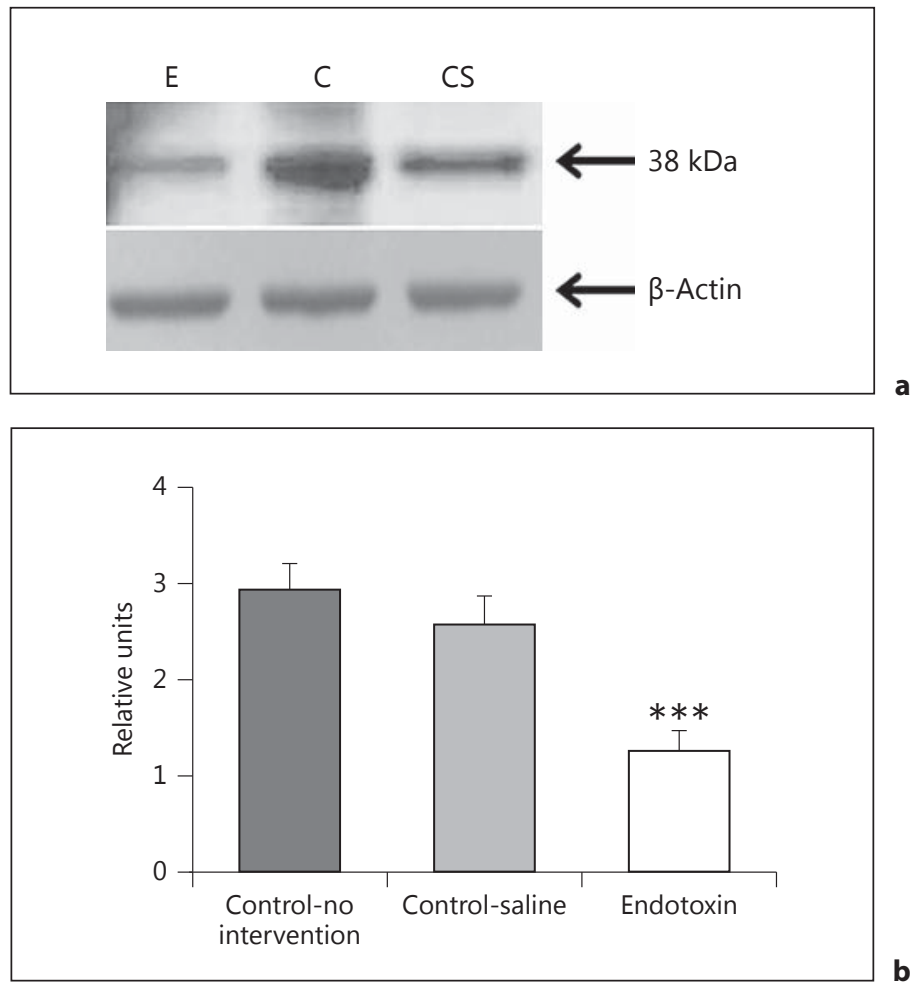

Fig. 5. Synaptophysin expression is decreased in the cortex of endotoxin-exposed kits. a Immunoblot analysis of synaptophysin expression in the cortex of endotoxin-exposed (E), control-no intervention (C), and control-saline (CS) kits. $\beta$-Actin was used as a loading control. b Densitometric analysis revealed that the synaptophysin expression was significantly less in the endotoxin-exposed kits than in the two control groups. ${ }^{* * *} \mathrm{p}<0.001$, endotoxin vs. control groups (5 kits/group).

concordance with our hypothesis, the Western blot analysis demonstrated that synaptophysin expression was significantly reduced in the endotoxin group compared to that in the control-no intervention and control-saline groups ( $\mathrm{p}<0.001$; fig. 5).

\section{Discussion}

Our study demonstrates that maternal intrauterine inflammation in pregnant rabbits at gestational day 28 causes a reduction in the number of MAP2-stained neurons in the anterior thalamus of newborn kits. The decrease in anterior thalamic neurons was due to increased neurodegeneration, as evidenced by an increase in FluorJade C-positive cells in the thalamus. In addition, endotoxin-exposed kits had decreased dendritic arborization and spine density in the RSC, which receives input from 
the anterior thalamus and hippocampus. Activated microglia are known to cause injury to neurons through formation of free radicals, pro-inflammatory cytokines and excitotoxic metabolites $[35,36]$. Although a robust increase in activated microglia in the white matter region of the brain is characteristic in our model, presence of activated microglia in the anterior thalamic nuclei was not observed. However, output from the thalamus traverses the white matter tracts which are infiltrated with activated microglia. Injury to the neurons may occur when they migrate through these white matter tracts [37]. However, neurons that form the anterior thalamic group in the rabbits originate between the 15th and 18th day of gestation, a time that precedes the introduction of LPS in our model [38]. Thus, the loss of neurons in the anterior thalamus may be secondary to axonal injury and impaired axonal transport, which leads to retrograde neuronal degeneration. A recent study has shown that glutamatergic neurogenesis continues in the rabbit brain even at gestational day 32 [39]. It is also possible that the decrease in the number of thalamic neurons observed in this study may be partly attributed to a decrease in neuronal progenitors in the subventricular zone.

Numerous studies have shown that intrauterine inflammation can contribute to preterm birth and subsequent developmental abnormalities [37, 40-42]. Burd et al. $[41,42]$ have reported that maternal inflammation-induced premature delivery is associated with a decrease in MAP2-stained neurons and a decrease in dendritic arbor in cortical neuronal cultures. They suggested that the presence of proinflammatory cytokines may alter neuronal morphology, leading to a spectrum of cognitive and neurobehavioral disorders. Maternal LPS injection has been shown to alter cytokine levels and the profile of cortical neurons in neuronal cultures from mouse pups at term [43]. Additionally, abnormal dendritic arborization in the medial prefrontal cortex and hippocampus of neonatal and adult rats has been reported in an inflammatory model of schizophrenia [40]. These reports emphasize that injury to cortical neurons after maternal infection occurs in both preterm and term offspring. Our own results, taken together with these previous findings, highlight the importance of maternal inflammation in altering neuronal architecture in the neonatal brain. In our study, we found that along with the loss of dendritic arborization, there is a loss of the thalamic neurons that provide input to the RSC.

Dendritic branches are dynamic integrators of synaptic information and are essential for normal neuronal functioning. The number of branches or the branching pattern of neurons highly influences the transmission of

Maternal Inflammation and Neuronal Injury in Rabbit Kits synaptic signals to the soma; thus, a decrease in dendritic branches may hinder processing of neuronal information [44]. The decrease in dendritic arborization and spine density that we observed may also be due to a decrease in serotonin in the cortex. We have previously reported that maternal intrauterine inflammation and microglial activation in the fetal/neonatal brain results in decreased metabolism of tryptophan to serotonin in the cortex of newborn kits [12]. Serotonin is required for neurogenesis, neuronal migration, and synaptogenesis $[45,46]$. Therefore, decreased serotonin can alter the dendritic arborization and perturb the developmental process in the cerebral cortex and hippocampus [47-49].

In our animal model, we observed a decrease in the spine density and synaptophysin expression in the newborn brain after maternal intrauterine LPS administration. Spines are highly dynamic structures that harbor most of the excitatory synapses and consist of receptors and proteins that help in synaptic transmission [50]. Loss of spines has been reported in a number of neuroinflammatory disorders, such as Alzheimer disease, schizophrenia, and Parkinson disease [51]. The loss of spine density that we observed may result in decreased relay of inputs to RSC neurons, which may in turn influence the arborization of the RSC neurons.

Synaptophysin is a presynaptic terminal vesicle protein that is synthesized in the soma and transported anterograde via the axon. This vesicle protein is involved in exocytosis, synapse formation and regulating synaptic transmission in circuits involving learning and memory [52-55]. Although the loss of thalamic neurons may result in decreased expression of synaptophysin in the cortex, activated microglia have also been reported to inhibit the axonal transport of synaptophysin directly [56]. We have previously demonstrated robust microglial activation in the periventricular regions of newborn rabbits exposed to maternal inflammation $[10,11]$. The decreased synaptophysin expression in the cortex of kits exposed to maternal intrauterine inflammation may be caused by loss of thalamic neurons or the direct action of activated microglia on synaptophysin transport. A decreased level of synaptophysin has also been associated with loss of spines in other neuroinflammatory disease such as Alzheimer's disease [57]. Thus, the reduction in number of branches and spines in the retrosplenial cortical neurons observed in our study would parallel the loss of presynaptic terminals arising from thalamic neurons.

Lesions of the anterior thalamus are known to result in a loss of synaptic plasticity in the RSC [21]. A loss of neurons in the thalamus has been reported in patients with 
PVL [6]. Similarly, increased apoptosis of anterior thalamic neurons was observed in a rabbit model of fetal hypoxia-ischemia-induced CP [30]. Increased neuronal degeneration and inflammation were also noted in the periventricular region in a rabbit model of intraventricular hemorrhage-induced brain injury $[39,58]$. In our study, the loss of neurons in the anterior thalamus was associated with a decrease in dendritic arborization and spine density in the RSC of newborn rabbit kits following intrauterine endotoxin exposure. Because the RSC receives its major input from the anterior thalamus and is also involved in cognitive function, it is possible that the loss of dendritic profile in the RSC may result from loss of target neurons. Thus, loss of anterior thalamic neurons along with an abnormal neuronal profile in the RSC may provide the basis for cognitive deficits in patients with $\mathrm{CP}$.

\section{Conclusion}

Behavioral and cognitive disorders are seen in 30$50 \%$ of patients with CP [59]. Up to $59 \%$ of patients with PVL have thalamic damage that may explain the cogni- tive deficits often seen in CP [6]. The loss of thalamic neurons and loss of dendrites and spines in the RSC that we have reported in this study may explain the high incidence of behavioral and cognitive impairments associated with perinatal brain injury. Additional studies will be needed to help determine if rabbit kits exposed to maternal endotoxin administration develop cognitive deficits. Since there is a greater incidence of CP in males compared to females [60], future studies may be warranted to evaluate the effect of sex on the extent of neuronal injury after maternal intrauterine infection/inflammation.

\section{Acknowledgements}

This study was supported in part by 1K08HD050652, R01 HD069562, NICHD, NIH, and the Perinatology Research Branch, Eunice Kennedy Shriver NICHD, NIH, DHHS.

We would like to thank Dr. Steven Di Carlo for the use of his Stereo Investigator.

\section{References}

1 Yoon BH, Romero R, Park JS, Kim CJ, Kim SH, Choi JH, Han TR: Fetal exposure to an intraamniotic inflammation and the development of cerebral palsy at the age of three years. Am J Obstet Gynecol 2000;182:675-681.

-2 Vargas DL, Nascimbene C, Krishnan C, Zimmerman AW, Pardo CA: Neuroglial activation and neuroinflammation in the brain of patients with autism. Ann Neurol 2005;57:67-81.

$\checkmark 3$ Patterson PH: Immune involvement in schizophrenia and autism: etiology, pathology and animal models. Behav Brain Res 2009; 204:313-321.

-4 Andiman SE, Haynes RL, Trachtenberg FL, Billiards SS, Folkerth RD, Volpe JJ, Kinney HC: The cerebral cortex overlying periventricular leukomalacia: analysis of pyramidal neurons. Brain Pathol 2010;20:803-814.

5 Banker BQ, Larroche JC: Periventricular leukomalacia of infancy. A form of neonatal anoxic encephalopathy. Arch Neurol 1962; 386-410.

6 Ligam P, Haynes RL, Folkerth RD, Liu L, Yang M, Volpe JJ, Kinney HC: Thalamic damage in periventricular leukomalacia: novel pathologic observations relevant to cognitive deficits in survivors of prematurity. Pediatr Res 2009;65:524-529.
-7 Pierson CR, Folkerth RD, Billiards SS, Trachtenberg FL, Drinkwater ME, Volpe JJ, Kinney HC: Gray matter injury associated with periventricular leukomalacia in the premature infant. Acta Neuropathol 2007;114: 619-631.

8 Billiards SS, Haynes RL, Folkerth RD, Trachtenberg FL, Liu LG, Volpe JJ, Kinney HC: Development of microglia in the cerebral white matter of the human fetus and infant. J Comp Neurol 2006;497:199-208.

-9 Hagberg H, Gressens P, Mallard C: Inflammation during fetal and neonatal life: implications for neurologic and neuropsychiatric disease in children and adults. Ann Neurol 2012; 71:444-457.

10 Kannan S, Saadani-Makki F, Balakrishnan B, Chakraborty P, Janisse J, Lu X, Muzik O, Romero R, Chugani DC: Magnitude of [(11) C]PK11195 binding is related to severity of motor deficits in a rabbit model of cerebral palsy induced by intrauterine endotoxin exposure. Dev Neurosci 2011;33:231-240.

-11 Kannan S, Saadani-Makki F, Muzik O, Chakraborty P, Mangner TJ, Janisse J, Romero R, Chugani DC: Microglial activation in perinatal rabbit brain induced by intrauterine inflammation: detection with 11C-(R)PK11195 and small-animal PET. J Nucl Med 2007;48:946-954.
-12 Kannan S, Saadani-Makki F, Balakrishnan B, Dai H, Chakraborty PK, Janisse J, Muzik O, Romero R, Chugani DC: Decreased cortical serotonin in neonatal rabbits exposed to endotoxin in utero. J Cereb Blood Flow Metab 2011;31:738-749.

13 Gaspar P, Cases O, Maroteaux L: The developmental role of serotonin: news from mouse molecular genetics. Nat Rev Neurosci 2003;4: 1002-1012.

14 Yan W, Wilson CC, Haring JH: Effects of neonatal serotonin depletion on the development of rat dentate granule cells. Brain Res Dev Brain Res 1997;98:177-184.

15 Yokochi K: Thalamic lesions revealed by MR associated with periventricular leukomalacia and clinical profiles of subjects. Acta Paediatr 1997;86:493-496.

16 Ricci D, Anker S, Cowan F, Pane M, Gallini F, Luciano R, Donvito V, Baranello G, Cesarini L, Bianco F, Rutherford M, Romagnoli C, Atkinson J, Braddick O, Guzzetta F, Mercuri E: Thalamic atrophy in infants with PVL and cerebral visual impairment. Early Hum Dev 2006;82:591-595.

17 Gimenez M, Junque C, Narberhaus A, Botet F, Bargallo N, Mercader JM: Correlations of thalamic reductions with verbal fluency impairment in those born prematurely. Neuroreport 2006;17:463-466. 
18 van GT, Wyss JM: Connections of the retrosplenial granular b cortex in the rat. J Comp Neurol 2003;463:249-263.

19 Vann SD, Aggleton JP, Maguire EA: What does the retrosplenial cortex do? Nat Rev Neurosci 2009;10:792-802.

-20 Fazio F, Perani D, Gilardi MC, Colombo F, Cappa SF, Vallar G, Bettinardi V, Paulesu E, Alberoni M, Bressi S: Metabolic impairment in human amnesia: a PET study of memory networks. J Cereb Blood Flow Metab 1992;12: 353-358.

21 Garden DL, Massey PV, Caruana DA, Johnson B, Warburton EC, Aggleton JP, Bashir ZI: Anterior thalamic lesions stop synaptic plasticity in retrosplenial cortex slices: expanding the pathology of diencephalic amnesia. Brain 2009;132:1847-1857.

-22 Nestor PJ, Fryer TD, Ikeda M, Hodges JR: Retrosplenial cortex (BA 29/30) hypometabolism in mild cognitive impairment (prodromal Alzheimer's disease). Eur J Neurosci 2003;18:2663-2667.

-23 Yasuda Y, Watanabe T, Tanaka H, Tadashi I, Akiguchi I: Amnesia following infarction in the right retrosplenial region. Clin Neurol Neurosurg 1997;99:102-105.

- 24 Valenstein E, Bowers D, Verfaellie M, Heilman KM, Day A, Watson RT: Retrosplenial amnesia. Brain 1987;110:1631-1646.

-25 Rudge P, Warrington EK: Selective impairment of memory and visual perception in splenial tumours. Brain 1991;114:349-360.

-26 Maguire EA: The retrosplenial contribution to human navigation: a review of lesion and neuroimaging findings. Scand J Psychol 2001; 42:225-238.

-27 Saadani-Makki F, Kannan S, Makki M, Muzik O, Janisse J, Romero R, Chugani D: Intrauterine endotoxin administration leads to white matter diffusivity changes in newborn rabbits. J Child Neurol 2009;24:1179-1189.

28 Saadani-Makki F, Kannan S, Lu X, Janisse J, Dawe E, Edwin S, Romero R, Chugani D: Intrauterine administration of endotoxin leads to motor deficits in a rabbit model: a link between prenatal infection and cerebral palsy. Am J Obstet Gynecol 2008;199:651-657.

-29 Kannan S, Dai H, Navath RS, Balakrishnan B, Jyoti A, Janisse J, Romero R, Kannan RM: Dendrimer-based postnatal therapy for neuroinflammation and cerebral palsy in a rabbit model. Sci Transl Med 2012;18:130-146.

-30 Derrick M, Luo NL, Bregman JC, Jilling T, Ji X, Fisher K, Gladson CL, Beardsley DJ, Murdoch G, Back SA, Tan S: Preterm fetal hypoxia-ischemia causes hypertonia and motor deficits in the neonatal rabbit: a model for human cerebral palsy? J Neurosci 2004;24:24-34.

- 31 Gundersen HJ, Bendtsen TF, Korbo L, Marcussen N, Moller A, Nielsen K, Nyengaard JR, Pakkenberg B, Sorensen FB, Vesterby A, et al: Some new, simple and efficient stereological methods and their use in pathological research and diagnosis. APMIS 1988;96:379394.
32 Shek JW, Wen GY, Wisniewski HM: Atlas of the Rabbit Brain and Spinal Cord. Basel, Karger, 1986.

33 Sholl DA: Dendritic organization in the neurons of the visual and motor cortices of the cat. J Anat 1953;87:387-406.

34 Takamori S, Holt M, Stenius K, Lemke EA, Gronborg M, Riedel D, Urlaub H, Schenck S, Brugger B, Ringler P, et al: Molecular anatomy of a trafficking organelle. Cell 2006;127: 831-846.

35 Block ML, Hong JS: Chronic microglial activation and progressive dopaminergic neurotoxicity. Biochem Soc Trans 2007;35:11271132.

36 Girard S, Kadhim H, Roy M, Lavoie K, Brochu ME, Larouche A, Sebire G: Role of perinatal inflammation in cerebral palsy. Pediatr Neurol 2009;40:168-174.

37 Leviton A, Gressens P: Neuronal damage accompanies perinatal white-matter damage. Trends Neurosci 2007;30:473-478.

38 Fernandez V: An autoradiographic study of the development of the anterior thalamic group and limbic cortex in the rabbit. J Comp Neurol 1969;136:423-451.

-39 Malik S, Vinukonda G, Vose LR, Diamond D, Bhimavarapu BB, Hu F, Zia MT, Hevner R, Zecevic N, Ballabh P: Neurogenesis continues in the third trimester of pregnancy and is suppressed by premature birth. J Neurosci 2013; 33:411-423.

40 Baharnoori M, Brake WG, Srivastava LK: Prenatal immune challenge induces developmental changes in the morphology of pyramidal neurons of the prefrontal cortex and hippocampus in rats. Schizophr Res 2009;107: 99-109.

41 Burd I, Bentz AI, Chai J, Gonzalez J, Monnerie H, Le Roux PD, Cohen AS, Yudkoff M, Elovitz MA: Inflammation-induced preterm birth alters neuronal morphology in the mouse fetal brain. J Neurosci Res 2010;88: 1872-1881.

42 Burd I, Chai J, Gonzalez J, Ofori E, Monnerie H, Le Roux PD, Elovitz MA: Beyond white matter damage: fetal neuronal injury in a mouse model of preterm birth. Am J Obstet Gynecol 2009;201:279-278.

43 Elovitz MA, Brown AG, Breen K, Anton L, Maubert M, Burd I: Intrauterine inflammation, insufficient to induce parturition, still evokes fetal and neonatal brain injury. Int J Dev Neurosci 2011;29:663-671.

44 Hausser M, Spruston N, Stuart GJ: Diversity and dynamics of dendritic signaling. Science 2000;290:739-744.

45 Lauder JM, Krebs H: Serotonin as a differentiation signal in early neurogenesis. Dev Neurosci 1978;1:15-30.

46 Mazer C, Muneyyirci J, Taheny K, Raio N, Borella A, Whitaker-Azmitia P: Serotonin depletion during synaptogenesis leads to decreased synaptic density and learning deficits in the adult rat: a possible model of neurodevelopmental disorders with cognitive deficits. Brain Res 1997;760:68-73.
47 Whitaker-Azmitia PM, Borella A, Raio N: Serotonin depletion in the adult rat causes loss of the dendritic marker MAP-2. A new animal model of schizophrenia? Neuropsychopharmacology 1995;12:269-272.

48 Durig J, Hornung JP: Neonatal serotonin depletion affects developing and mature mouse cortical neurons. Neuroreport 2000;11:833837.

49 Bonnin A, Levitt P: Fetal, maternal, and placental sources of serotonin and new implications for developmental programming of the brain. Neuroscience 2011;197:1-7.

50 Kennedy MB: Signal-processing machines at the postsynaptic density. Science 2000;290: 750-754.

$51 \mathrm{Yu}$ W, Lu B: Synapses and dendritic spines as pathogenic targets in Alzheimer's disease. Neural Plast 2012;2012:247150.

52 Leube RE, Wiedenmann B, Franke WW: Topogenesis and sorting of synaptophysin: synthesis of a synaptic vesicle protein from a gene transfected into nonneuroendocrine cells. Cell 1989;59:433-446.

53 Schmitt U, Tanimoto N, Seeliger M, Schaeffel F, Leube RE: Detection of behavioral alterations and learning deficits in mice lacking synaptophysin. Neuroscience 2009;162:234243.

54 Tarsa L, Goda Y: Synaptophysin regulates activity-dependent synapse formation in cultured hippocampal neurons. PNAS 2002;99: 1012-1016.

55 Tarpey PS, Smith R, Pleasance E, Whibley A, Edkins S, Hardy C, et al: A systematic, largescale resequencing screen of $\mathrm{X}$-chromosome coding exons in mental retardation. Nat Genet 2009;41:535-543.

56 Stagi M, Dittrich PS, Frank N, Iliev AI, Schwille P, Neumann H: Breakdown of axonal synaptic vesicle precursor transport by microglial nitric oxide. J Neurosci 2005;25: 352-362.

-57 Spires TL, Meyer-Luehmann M, Stern EA, McLean PJ, Skoch J, Nguyen PT, et al: Dendritic spine abnormalities in amyloid precursor protein transgenic mice demonstrated by gene transfer and intravital multiphoton microscopy. J Neurosci 2005;25:7278-7287.

58 Vinukonda G, Csiszar A, Hu F, Dummula K, Pandey NK, Zia MT, Ferreri NR, Ungvari Z, LaGamma EF, Ballabh P: Neuroprotection in a rabbit model of intraventricular haemorrhage by cyclooxygenase-2, prostanoid receptor-1 or tumour necrosis factor-alpha inhibition. Brain 2010;133:2264-2280.

59 Marlow N, Wolke D, Bracewell M, Samara M: Neurologic and developmental disability at six years after extremely preterm birth. N Engl J Med 2005;352:9-19.

60 Arneson CL, Durkin MS, Benedict RE, Kirby RS, Yeargin-Allsopp M, Van Naarden Braun K, Doernberg NS: Prevalence of cerebral palsy: Autism and Developmental Disabilities Monitoring Network, three sites, United States, 2004. Disabil Health J 2009;2:45-48. 